\title{
MULTI-SCALE CREST LINE EXTRACTION BASED ON HALF GAUSSIAN KERNELS
}

\author{
Baptiste Magnier ${ }^{\star}$, Arezki Aberkane ${ }^{\star}$, Philippe Borianne ${ }^{\odot}$, Philippe Montesinos $^{\star}$ and Christophe Jourdan ${ }^{\otimes}$

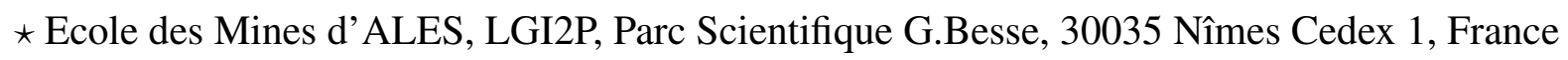 \\ $\odot$ CIRAD, Boulevard de la Lironde, TA A-51 / PS2 34398, Montpellier Cedex 5, France \\ $\otimes$ UMR Eco \& Sols, CIRAD, 2 place Viala, 34060 Montpellier, France
}

\begin{abstract}
Crest line extraction has always been a challenging task in image processing and its applications. It is possible to detect ridges and valleys in images using second order filters. In order to estimate crest lines of variable widths, a multiscale analysis of the image is required. In this paper we propose a new ridge/valley detection method in images based on the difference of rotating Gaussian semi filters adapted in a multi-scale process. Due to the directional filters, we obtain a new ridge/valley anisotropic detector enabling very precise detection of ridge/valley of varied widths. Moreover, as the detector filters compute the two directions of crest lines, even highly bended crest lines are correctly extracted. Numerical comparisons with other oriented Gaussian filters and results on real images validate the interest of this method.
\end{abstract}

Index Terms- Crest lines, multi-scale, steerable filter.

\section{CREST LINE EXTRACTION}

Crest lines or curves in an image represent narrow ridges or valleys in the image surface. They may define thin nets inside an image describing roads or rivers in satellite images, blood vessels in medical images or roots in underground images. Therefore, finding these dense and thin structures is an important issue in image processing.

In a grey level image $I$, ridges are maxima, valleys are the minima [1]. Indeed, considering the image surface $S$, in the cartesian space, the following equation defines all pixel coordinates: $\vec{S}(x, y)=(x, y, I(x, y))^{t}$. Let $S_{T}(x, y)$ be the tangential plane to the surface in all points where:

$$
S_{T}(x, y)=\left\{\left(1,0, I_{x}(x, y)\right)^{t},\left(0,1, I_{y}(x, y)\right)^{t}\right\},
$$

with $\left(I_{x}, I_{y}\right)$ the partial derivatives of $I$ respectively along the $x$ and the $y$ axis. Ridges and valleys are given by the points where the values of $\vec{S}(x, y)$ are maxima or, respectively, minima in the orthogonal direction to the curve at $(x, y)$.

The literature presents several methods for ridges and valleys detection. In image filtering, convolving $I$ with the second derivative of the Gaussian filter in two dimensions, and then removing the non local maxima in the direction maximizing the variance allows a detection of crest lines [2]. Also, the Hessian matrix consists of the second partial derivatives of the image after convolving with a Gaussian smoothing kernel. Computation of the orthogonal direction of a curve for a pixel can be tackled by finding the eigenvector that corresponds to the maximum absolute eigenvalue of the Hessian matrix [3] [4] [5] or the Weingarten endomorphism matrix [6] of the considered pixel. However, results obtained by these approaches can present increased false detection rate in noisy images. It is mainly because the high pass filtering used for the second derivative is sensitive to the noise level.

In order to detect oriented contours or ridges, Freeman and Adelson proposed to use oriented Gaussian derivative kernels called steerable filters [7]. Thus, the second order oriented Gaussian becomes a crest line detector as illustrated in Fig. 1(a). As represented in Fig. 1(c), the second partial derivative of anisotropic Gaussian kernels introduced by Perona [8] constitutes a good estimator of ridges and valleys (note that the Difference of Oriented Gaussian (DOG) with a standard deviation ratio of 1.6 allows a crest lines estimation because it approximates well a second derivate of Gaussian (Laplacian of Gaussian -LoG- [9] [10]). These oriented kernels characterize the pixels belonging to a contour by the fact that their oriented energy is maximum in the orthogonal direction to their local orientation. Afterward, Jacob and Unser [11] have developed special families of edges and ridges detectors based on Gaussian derivatives which are derived from the criteria used by Canny to design his famous edge detector [12]. In particular, second and forth order Gaussian derivatives are presented for the extraction of crest lines (Fig. 1). These criteria are expressed under an integral form and assembled into a quadratic criterion, whose minimization with a Lagrange multiplier method leads to an eigenvalue calculus. These approaches are able to correctly detect large oriented

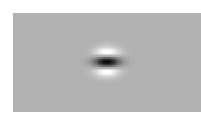

(a) Isotropic Gaussian, order 2

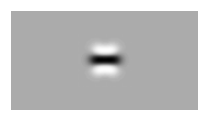

(b) Isotropic Gaussian, order 4

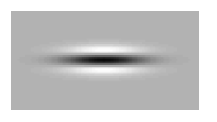

(c) Anisotropic Gaussian kernel

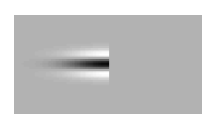

(d) Half anisotropic Gaussian kernel
Fig. 1. Different $2 D$ crest line detectors. For (c) and (d) are used the following ratios: $\lambda_{2} / \lambda_{1}=1.6$ and $\mu / \lambda_{1}=5$. 
linear structures like straight lines. For anisotropic filtering methods [8], the robustness against noise depends strongly on the two smoothing parameters of the filter (length and width). If these parameters increase, the detection is less sensitive to noise, but thin crest lines are considered as noise, and, as for the conventional crest line detectors, the estimated position of the line moves farther away from the true position. Moreover, as shown in Fig. 3 (b), junctions and corners are not well detected with these different methods [13]. Indeed, the detector value is greater near the corner than on the corner itself [14]. Consequently, the precision of the detected ridge or valley points decreases strongly at corner points and for non straight object contour parts.

Based on difference of two half Gaussian smoothing kernels, a new anisotropic crest line detection method has been developed in [14]. This approach is also able to detect junctions and corners thanks to elongated and oriented filters in two different directions (see Fig. 2). Therefore, smoothing with rotating filters means that the image is smoothed with a bank of rotated half anisotropic Gaussian kernels [15] [16]:

$$
G_{(\mu, \lambda)}(x, y)=\frac{1}{2 \pi \lambda \mu} \cdot H_{y}\left(e^{-\frac{x^{2}}{2 \cdot \lambda^{2}}-\frac{y^{2}}{2 \cdot \mu^{2}}}\right),
$$

where $(x, y)$ are pixel coordinates and $(\mu, \lambda)$ the standarddeviations of the Gaussian filter. Contrary to [8], as we need only the causal part of the filter (illustrated on Fig. 1(d) and 2), we simply "cut" the smoothing kernel by the middle, this operation corresponds to the Heaviside function $H_{y}$ along the $\mathrm{y}$ axis [15]. Using a discrete rotation $R_{\theta}$ of angle $\theta$ of the original image $I$, by convolution [17], we obtain a collection of directional smoothed images $I_{\theta}=\left(R_{\theta} \cdot I\right) * G_{(\mu, \lambda)}$. In a second step, we apply an inverse rotation of the smoothed image and obtain a bank of $360^{\circ} / \Delta \theta$ where $\Delta \theta$ represents the step between each discretized orientation. This stage is repeated twice with filters of different widths but of the same length before applying the difference: $D(x, y, \theta)=G_{\left(\mu, \lambda_{1}\right)}(x, y)-$ $G_{\left(\mu, \lambda_{2}\right)}(x, y)$, with $\lambda_{2} / \lambda_{1}=1.6$ [9] [10]. Thus, we obtain for each pixel a signal $D(x, y, \theta)$ which corresponds to a scan in $360 / \Delta \theta$ directions [14]. Then a ridge/valley operator $\Sigma_{\left(\mu, \lambda_{1}\right)}(x, y)$ is defined as:

$$
\begin{aligned}
\Sigma_{\left(\mu, \lambda_{1}\right)}(x, y)=\quad & D\left(x, y, \theta_{M_{1}}\right)+D\left(x, y, \theta_{M_{2}}\right) \\
+ & D\left(x, y, \theta_{m_{1}}\right)+D\left(x, y, \theta_{m_{2}}\right)
\end{aligned}
$$

where $\theta_{M_{1}}, \theta_{M_{2}}$ are the directions of the local maxima of the function $D$ (see Fig. 2) and $\theta_{m_{1}}, \theta_{m_{2}}$ the directions of the local minima. Conditions of detection are as follows:

if $\Sigma_{\left(\mu, \lambda_{1}\right)}(x, y)>\Sigma_{t h}$, the pixel P belongs to a ridge,

if $\Sigma_{\left(\mu, \lambda_{1}\right)}(x, y)<-\Sigma_{t h}$, the pixel P belongs to a valley, where $\Sigma_{t h}>0$. Finally, ridges can easily be extracted computing local maxima of $\Sigma_{\left(\mu, \lambda_{1}\right)}(x, y)$ in the $\eta$ direction, bisector of $\left(\theta_{M_{1}}, \theta_{M_{2}}\right)$ and valleys computing the minima of $\Sigma_{\left(\mu, \lambda_{1}\right)}(x, y)$ in the $\eta$ direction, bisector of $\left(\theta_{m_{1}}, \theta_{m_{2}}\right)$ (see Fig. 2). Thereafter is developed the method in a multi-scale approach.
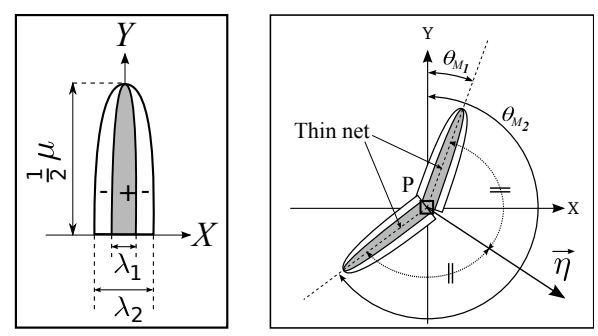

Fig. 2. Difference of half rotating smoothing kernel description and parameters. It computes the two directions of a curve even if they are discontinuous or highly bended.

\section{MULTI-SCALE THIN NET EXTRACTION}

\subsection{Detection of Crest Lines of Different Widths}

Crest lines represent narrow objects in an image, however, these objects happen to have different widths. Thus, secondorder derivatives techniques can be commonly implemented in a multi-scale framework to detect ridges or valleys of different sizes. The choice and number of scales is especially important since too small scales emphasize small details, while producing spurious responses to noise or texture, tangent objects and other features. Otherwise, too large scales deform original objects at detection rounding corners and by loosing details. Scale selection is most often performed by choosing the maximum principal curvature of the image surface among the Gaussian kernels of different scale parameters. According to a function depending on the scale, it is possible to weight the second-order Gaussian filter responses such that this weighted response is maximized when the true scale of the structure is present. In the literature, various measures of crest lines strength have been proposed concerning isotropic Gaussian filters [3] [6]. Oriented Gaussian filters [7] [8] [11] can be performed in multi-scale framework in order to detect objects of different sizes. Thus, at each pixel of coordinates $(x, y)$, the selected scale $\mathbf{W}(x, y)$ is calculated as the maximum response of the oriented operator $F_{\theta_{i}, \sigma_{j}}(x, y)$, an oriented filter response, at the orientation $\theta_{\mathbf{W}}(x, y)$ for the discrete sampling of the angle and the scale domain [18]:

$$
\left\{\begin{aligned}
\mathbf{W}(x, y) & =\max _{j}\left(\max _{i}\left(F_{\theta_{i}, \sigma_{j}}(x, y)\right)\right), \\
\theta_{\mathbf{W}}(x, y) & =\underset{\theta_{i}(x, y)}{\arg \max }\left(F_{\theta_{i}, \sigma_{j}}(x, y)\right),
\end{aligned}\right.
$$

where $\theta_{i}(x, y)$ represents the $i^{\text {th }}$ discrete orientation of the filter at a scale $\sigma_{j}$ maximizing $F_{\theta_{i}, \sigma_{j}}(x, y)$. As seen in the previous section, $\sigma_{j}=\lambda_{1}$ for anisotropic DOG filter. Then, crest lines can be extracted computing local maxima of $\mathbf{W}(x, y)$ in the direction perpendicular to $\theta_{\mathbf{W}}(x, y)$. These different multi-scale techniques, isotropic [3] [6] [7] [8] [11] or anisotropic [18] suffer from the problem pointed out in the previous section i.e. they all fail at level of corners. 
Based on the analysis of the image singularities, a steerable, multi-scale singularity index for detecting crest lines has been developed [19]. As we will show in the next section, this approach, although robust against light noise, yields many false detections in textured or heavy noisy images.

\subsection{Multi-Scale Extraction of Crest Lines using Differ- ence of Oriented Half Gaussian Kernels}

In order to detect ridges and valleys of different sizes with their associated corners, being robust to noise and preventing false detection in presence of texture, we propose to adapt the last method based on half Gaussian kernels presented in Section 1 [14] in a multi-scale approach. On one hand, the ratio $\mu / \lambda_{1}$ must not be so large to enable the detection of bended and faint crest lines (illustrated in Fig. 3 (c - d)). On the other hand, a too short kernel is not robust against noise and does not extract correctly discontinuous lines in the image [14]. For that purpose, fixing $\mu / \lambda_{1}=5$, preserving the ratio $\lambda_{2} / \lambda_{1}=1.6$ (diagrammed in Fig. 4(a)), combining eq. 3 and eq. 4 , eventually noting $\Sigma_{\left(\mu, \lambda_{1}\right)}(x, y)=F_{\theta_{i}, \lambda_{1}}(x, y)$, we achieve a multi-scale analysis of the image, computing the maximum response of the anisotropic half Gaussian oriented filter with different scale parameters. Note that in eq. 4, $\eta(x, y)$ replaces $\theta_{i}$. Thus, from eq. 4 and $\eta(x, y)$, crest lines can easily be extracted computing local maxima of $\mathbf{W}(x, y)$ in the direction $\eta(x, y)$ followed by a hysteresis operator.

In a pixel precision process, as shown in [6], the optimal scale of the crest line's width called $w$ (i.e. the number of pixels) is $\lambda_{1}=w /(2 \sqrt{3})$. The smallest crest line width corresponds to one pixel, but Shannon's theorem states that the sampling frequency must be greater than twice the highest frequency contained in the signal, so the smallest starting scale corresponds to $\lambda_{1}=0.58$. Then, we can fix the scale variation by $\Delta \lambda_{1}=1 /(2 \sqrt{3}) \approx 0.29$.

\section{EVALUATION, RESULTS AND CONCLUSION}

\subsection{Evaluation and Comparison}

In order to carry out some quantitative results, we have conducted a number of tests using multi-scale filters with an image of wheat root system including valleys of different widths and sizes. The binary image in Fig. 4 (a) contains normally only one connected component corresponding to the root system. The small regions disconnected from the greater are
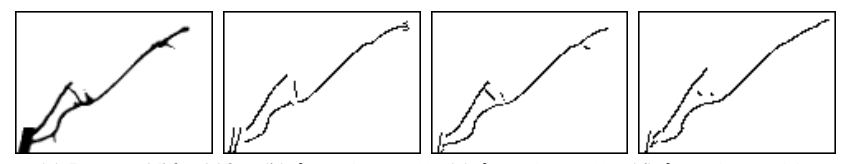

$\begin{array}{llll}\text { (a) Image, } 156 \times 112 & \text { (b) } \lambda_{1}=1, \mu=5 & \text { (c) } \lambda_{1}=1, \mu=5 & \text { (d) } \lambda_{1}=1, \mu=10\end{array}$

Fig. 3. Crest line extraction using: (b) full anisotropic Gaussian kernels and (c - d) half Gaussian kernels. suppressed. The (remaining) connected component is skeletonised, i.e. reduced to single-pixel-wide shapes by a wellknown thinning algorithm [20]. The skeleton is the almost8-connected-pixel line of local maxima of the distance-tocomponent-border function. The dissimilarity between two pixel lines - skeleton, ridge lines, medial axis, etc - is given by the sum of their differences, i.e. the number of pixels of the first line which are not in the second. The pixel-to-pixel comparison is done in a $3 \times 3$ neighborhood centered successively on the position of the first line pixels. For each point $(i, j)$ of the first line must find a point of the second in the $3 \times 3$ neighborhood centered on the $(i, j)$ position. The dissimilarity is increased by one each time no point is found. The introduction of neighborhood allows comparing lines with different pixel connectivity and small local variations. The dissimilarity factor is obtained dividing the counted differences by the number of the first line pixels.

In the case of the comparison between a $F$ line to a given $R$ reference line composed from $N$ pixels, the (factor of) dissimilarity allows evaluating the effectives of the true positive, false positive and false negative classes used in the performance [21] or sensitivity [22] functions which often characterize the produced results. The true positive are defined by the similarity between the two lines i.e. the complement to $N$ of the dissimilarity, the false positive (resp. false negative) by the dissimilarity between $R$ and $F$ (resp. $F$ and $R$ ).

With these functions, we analyzed the effect of the several multi-scale oriented crest line detectors on the reference image corrupted by a Gaussian noise, an uniform white noise or adding texture. The uniform noise and the texture are added on the original image (shown in Fig. 4) using the formula: $I_{m}=(1-L) \cdot I_{0}+L \cdot I_{N}$, where $I_{0}$ is the original image, $I_{N}$ an image of texture or random uniform noise and $I_{m}$ the resulting corrupted image.

Fig. 5 shows the relative variation of effectives of the class affecting the performance really; the results are obtained by

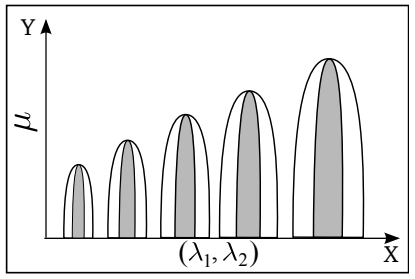

(a) Multi-scale half kernel evolution

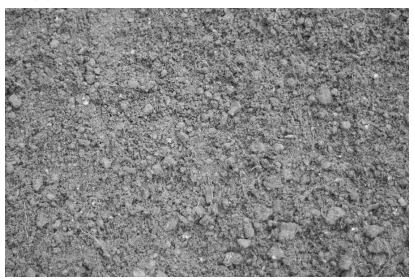

(c) Texture

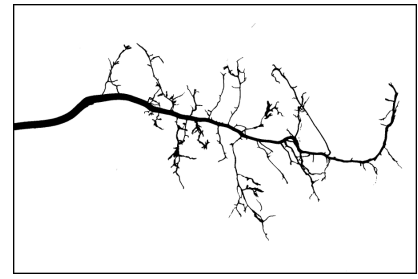

(b) Original image

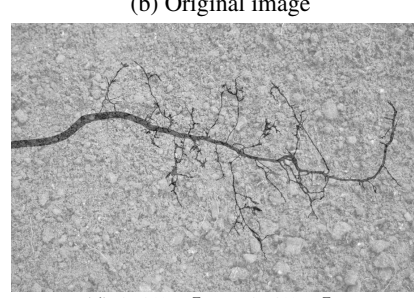

(d) $0.35 \cdot I_{0}+0.65 \cdot I_{N}$
Fig. 4. Addition of texture on a binarized image $600 \times 903$. 

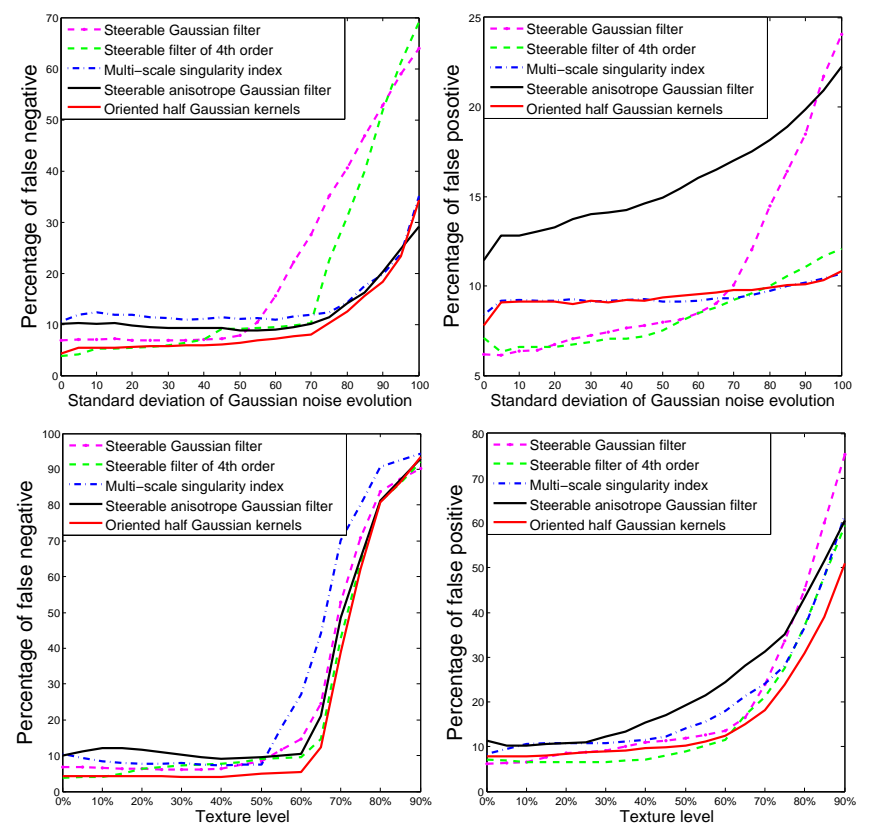

Fig. 5. Percentage error evaluation of image in Fig. 4(a).

several approaches [7] [8] [11] adapted in multi-scale (using 19 scales, see eq. 4 and section 2.2), our method and the multi-scale singularity index [19] in function of the texture level (i.e. the value of $L$ ) or the value of the Gaussian noise standard deviation. Evaluation of the different methods on an image corrupted by an uniform noise is similar with the addition of texture (multi-scale singularity index approach brings a little better result with uniform noise). Steerable Gaussian filters of order 2 or 4 achieved the best results when $I_{m}$ is not much corrupted, however, false negative and false positive points increase if the pollution becomes too heavy. Both of these methods produce interesting results in images containing small noise or texture level because they allow a detection of short crest lines, contrary to anisotropic filters which must have a standard deviation of filter's width five times smaller than its length (i.e. $\mu / \lambda_{1}=5$ ). Moreover, steerable anisotropic Gaussian filter shows a lot of false positive points, unlike half Gaussian filters which achieve always the less false negative points detection. Furthermore, the proposed method performs better in the presence of a heavy corruption of $I_{m}$ in terms of false positive point extraction.

\subsection{Result on Real Images and Conclusion}

In order to validate our approach, we have applied our multiscale filter on real images. Fig. 6(a) represents an image of a river delta captured by a NASA's standard spatial observer, here, we aim to extract all the arms of the river (the wide as the thin). We use 30 scales for each method adapted in multiscale [7] [8] [11] and ours. Steerable filter methods with full Gaussian kernels are not able to extract all the streams, espe-

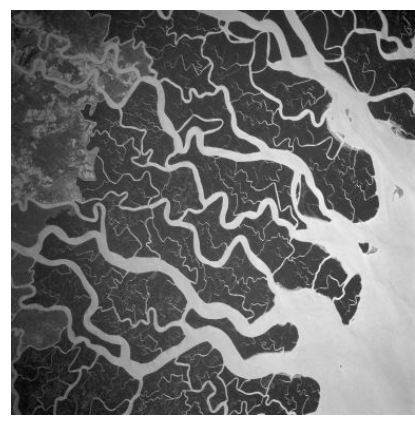

(a) Original image, $384 \times 384$

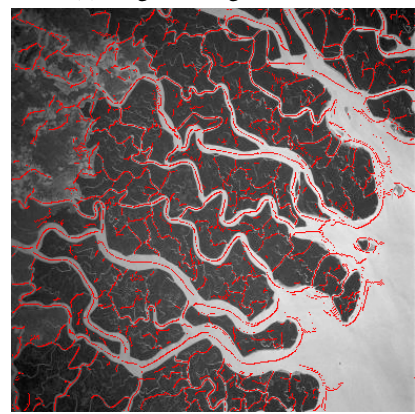

(c) Steerable filters of 4th order [11]

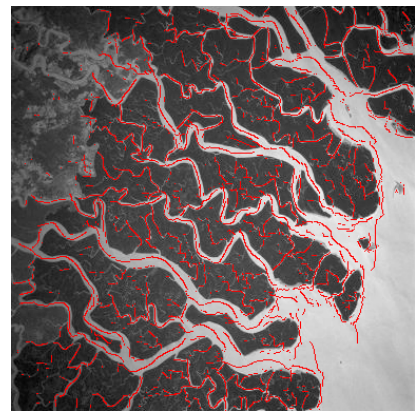

(e) Steerable anisotropic filters [8] [18]

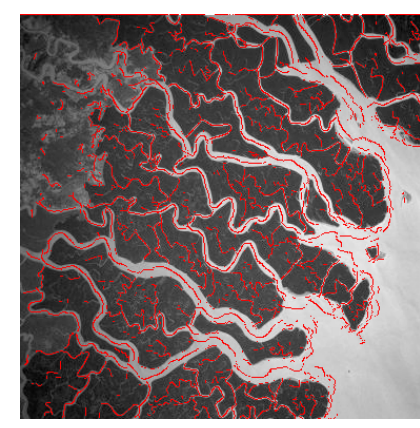

(b) Steerable filters of 2nd order [7]

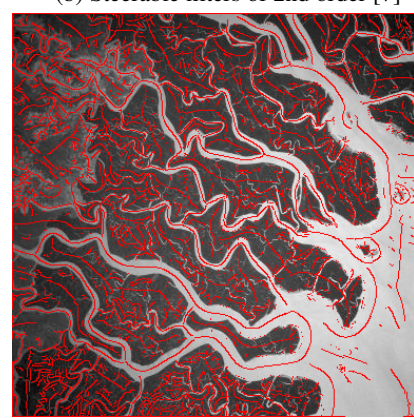

(d) Multi-scale singularity index [19]

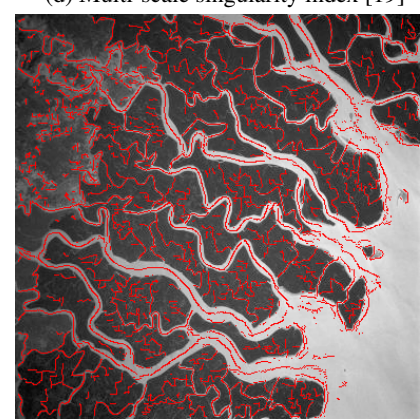

(f) Oriented half Gaussian kernels
Fig. 6. Multi-scale extraction of river delta.

cially when they are thin. The texture or the acquisition noise create false ridge detections for these methods. Our algorithm extracts almost all the different streams of all widths. For example, on Fig. 6(f), all the detected thin ridges, even bended, disappear under the red lines (i.e. under the detection).

This method obtains although promising results on large image databases of underground roots and retina medical images. Results on real images validate our method, which is a precise and a robust multi-scale detection approach of ridges and valleys. Next on our agenda is to adapt our method to 3D segmentation of blood vessel [23] and medical images [24].

\section{ACKNOWLEDGEMENTS}

The authors would like to thank the French Agence Nationale de la Recherche (project Ecosfix, ANR-2010-STRA-003) and the International Centre for Agricultural Research for Development (CIRAD) for providing the enabling environment and financial resources that allowed us to achieve this work. 


\section{REFERENCES}

[1] R. M. Haralick, "Ridges and valleys on digital images," Computer Vision, Graphics, and Image Processing, vol. 22 , no. 1 , pp. 28-38, 1983.

[2] D. Ziou, "Line detection using an optimal IIR filter," in Pattern Recognition, 1991, pp. 465-478.

[3] T. Lindeberg, "Edge detection and ridge detection with automatic scale selection," International Journal of Computer Vision, vol. 30, no. 2, pp. 117-154, 1998.

[4] C. Steger, "An unbiased detector of curvilinear structures," IEEE Transactions on Pattern Analysis and Machine Intelligence, vol. 20, no. 2, pp. 113-125, 1998.

[5] M. Cha, R. Phillips, and M. Yee, "Finding curves in sar ccd images," in IEEE International Conference on Acoustics, Speech, and Signal Processing 2011. IEEE, 2011, pp. 2024-2027.

[6] N. Armande, P. Montesinos, and O. Monga, "Thin nets extraction using a multi-scale approach," Scale-Space Theory in Computer Vision, pp. 361-364, 1997.

[7] W. T. Freeman and E. H. Adelson, "The design and use of steerable filters," IEEE Transactions on Pattern Analysis and Machine Intelligence, vol. 13, pp. 891906, 1991.

[8] P. Perona, "Steerable-scalable kernels for edge detection and junction analysis," Image and Vision Computing, vol. 10, no. 10, pp. 663-672, 1992.

[9] D. Marr and E. Hildreth, "Theory of edge detection," Proceedings of the Royal Society of London. Series B. Biological Sciences, vol. 207, no. 1167, pp. 187, 1980.

[10] T. Lindeberg, Scale-space theory in computer vision, Springer, 1993.

[11] M. Jacob and M. Unser, "Design of steerable filters for feature detection using canny-like criteria," IEEE Transactions on Pattern Analysis and Machine Intelligence, vol. 26, no. 8, pp. 1007-1019, 2004.

[12] F.J. Canny, "A computational approach to edge detection," IEEE Transactions on Pattern Analysis and Machine Intelligence, vol. 8, no. 6, pp. 679-698, 1986.

[13] B. Magnier, P. Montesinos, and D. Diep, "Ridge and valley junctions extraction," in International Conference on Image Processing, Computer Vision and Pattern Recognition (IPCV 2011), 2011.

[14] B. Magnier, D. Diep, and P. Montesinos, "Perceptual curve extraction," in IEEE 10th IVMSP (Image,
Video, and Multidimensional Signal Processing Technical Committee) on "Perception and Visual Signal Analysis" Workshop. IEEE, pp. 93-98.

[15] P. Montesinos and B. Magnier, "A new perceptual edge detector in color images," in Advanced Concepts for Intelligent Vision systems, 2010, pp. 209-220.

[16] B. Magnier, P. Montesinos, and D. Diep, "Texture removal by pixel classification using a rotating filter," in IEEE International Conference on Acoustics, Speech, and Signal Processing 2011, 2011, pp. 1097-1100.

[17] R. Deriche, "Recursively implementing the gaussian and its derivatives," in IEEE International Conference on Image Processing. A longer version is INRIA Research Report RR-1893, 1992, pp. 263-267.

[18] C. Sánchez, D. Gil, A. Rosell, A. Andaluz, and F. J. Sánchez, "Segmentation of tracheal rings in videobronchoscopy combining geometry and appearance," in International Conference on Computer Vision Theory and Application (VISAPP), 2013, pp. 153-161.

[19] G Muralidhar, A Bovik, and M Markey, "A steerable, multi-scale singularity index," IEEE Signal Processing Letter, vol. 20, no. 1, pp. 7-10, 2013.

[20] TY Zhang and Ching Y. Suen, "A fast parallel algorithm for thinning digital patterns," Communications of the $A C M$, vol. 27, no. 3, pp. 236-239, 1984.

[21] R. Pavez, M. Mora, and P. Gonzalez, "Objective comparison of contour detection in noisy images," in Progress in Pattern Recognition, Image Analysis, Computer Vision, and Applications, pp. 599-606. Springer, 2011.

[22] T. Fawcett, "An introduction to roc analysis," Pattern recognition letters, vol. 27, no. 8, pp. 861-874, 2006.

[23] N. Armande, P. Montesinos, and O. Monga, "A 3d thin nets extraction method for medical imaging," in Proceedings of the 13th International Conference on Pattern Recognition. IEEE, 1996, vol. 1, pp. 642-646.

[24] V Prinet, O Monga, C Ge, SL Xie, and SD Ma, "Thin network extraction in $3 \mathrm{~d}$ images: application to medical angiograms," in Proceedings of the 13th International Conference on Pattern Recognition. IEEE, 1996, vol. 3, pp. 386-390. 